


\title{
Alternativa didáctica interdisciplinar Para el PERFECCIONAMIENTO DE LA COMUNICACIÓN EDUCATIVA EN LA FORMACIÓN DE PROFESORES
}

\author{
ALTERNATIVE INTERDISCIPLINARY TEACHING FOR THE IMPROVEMENT OF
}

EDUCATIONAL COMMUNICATION IN TEACHER DEVELOPMENT

Dr.C. Ángel Arturo Leyva Silva, Ecuador - Universidad Tecnológica Israel, aleyva@uisrael.edu.ec. MsC. Ismary Gómez Díaz, Ecuador. Universidad Tecnológica Israel MsC..Elisa Sánchez Fereira, Cuba. Universidad "Camilo Cienfuegos",

Fecha de recepción: 12/05/2015

Fecha de aceptación: 28/06/2015

\begin{abstract}
Resumen
El trabajo fundamenta teórica y metodológicamente la necesidad y posibilidad de perfeccionar el proceso de enseñanza-aprendizaje en estudiantes universitarios en formación pedagógica. Se asume como fundamento para el trabajo el Enfoque Histórico-Cultural, y se analizan los conceptos "formación integral de la personalidad" y "comunicación" como mediadores en el proceso de asimilación del aprendizaje y la herencia cultural de la humanidad. El diagnóstico corrobora insuficiencias en la comunicación educativa y analiza las potencialidades para resolver los problemas, a través de una alternativa didáctica dirigida al perfeccionamiento de la comunicación educativa en la enseñanza de la didáctica especializada de la disciplina objeto de estudio.
\end{abstract}

Palabras claves: comunicación educativa, proceso de enseñanza aprendizaje, (PEA), formación pedagógica, Enfoque Histórico-Cultural. 


\section{Abstract}

This paper theoretically and methodologically lays the foundation for the necessity and possibility of improving the teaching-learning process in university students during the teacher training process, assuming as a basis the Historic-Cultural Approach. It analyzes the concepts "integral formation of personality" and "communication" as intermediaries in the learning assimilation process and cultural heritage of humanity. The diagnosis confirms inadequacies in the educational communication and analyzes the potential to solve problems through an educational alternative aimed at improving the educational communication in the teaching of specialized methodologies of the discipline being studied.

Keys Words: educational communication, teaching-learning process, teacher training, Historic-Cultural Approach. 


\section{Introducción}

La dinámica social y las exigencias del mundo profesional plantean constantemente exigencias cualitativas superiores al trabajo de los centros de Educación Superior en función de la calidad de la preparación de los futuros profesionales. Los egresados deben estar preparados para enfrentar los problemas concretos y prácticos que presenta a un país en vías de desarrollo y, fundamentalmente, deben ser competentes, a tenor con las futuras exigencias del desarrollo científico-técnico; siendo capaces de organizar y desarrollar la economía, la ciencia y la cultura de manera integral en bien de la sociedad.

Dentro de las vías para la preparación con calidad de los estudiantes de Ciencias Pedagógicas, se encuentra el perfeccionamiento del proceso de enseñanza-aprendizaje, (PEA) que propugne un aprendizaje desarrollador y constituya un modelo didáctico de cultura moderna de una docencia que rompa los esquemas tradicionales e ineficaces para tan significativa tarea.

Tal exigencia implica perfeccionar los métodos de enseñanza-aprendizaje y redeterminar las regularidades de la actividad cognoscitiva, de forma tal que se logre un salto en el aprendizaje teórico y práctico por parte de los estudiantes., lo que está condicionado por la transformación educacional que se produce en el mundo y las exigencias en la etapa actual, en función de la formación de los futuros profesionales de la educación, de manera tal que sean capaces de aplicar creadoramente los conocimientos para el desarrollo de la nueva sociedad.

Que el aprendizaje logre la independencia cognoscitiva en los estudiantes, es condición indispensable para la calidad del aprendizaje, aspecto que está condicionado por su vínculo con las necesidades, motivos e intereses del alumno. El profesor no es un mero trasmisor de conocimientos y el alumno un simple objeto receptor que única y pasivamente recibe influencias pedagógicas, tal como entiende la Pedagogía Tradicional, el profesor actual es un facilitador del proceso, que debe emplear una diversidad de métodos, medios y recursos que le permitan garantizar su propio desarrollo profesional y el de sus estudiantes.

Los autores de este trabajo, de vasta experiencia docentes, y después de la aplicación de varios instrumentos, han constatado que aún existen dificultades en el proceso de enseñanza-aprendizaje, las que atentan contra el estado deseado esbozado anteriormente.

Entre las dificultades existentes están las relacionadas con el hecho de que: 
- Los docentes se limitan a impartir conferencias, al estilo de la Pedagogía Tradicional, y en consecuencia a utilizar métodos de enseñanza-aprendizaje, que centran la actividad docente en ellos, en tal sentido, como consecuencia de lo planteado anteriormente se produce una sensible afectación de la comunicación alumno-profesor, creándose situaciones de incomunicación, en las cuales no se desarrollan habilidades de búsqueda y gestión del conocimiento por parte del alumno y al mismo tiempo de forma negativa se crea una falsa o aparente comunicación, en tanto, no se produce el intercambio alumno-profesor durante el proceso cognitivo, como exigencia y condición de una verdadera y eficiente comunicación educativa y formadora.

- Se obvian además las ventajas que ofrece el trabajo colaborativo y las posibilidades del establecimiento de relaciones ventajosas de comunicación.

- No se prioriza el intercambio grupal, la problematización de la enseñanza; y se desaprovechan así, sus potencialidades para el trabajo comunicativo.

- La orientación de la actividad independiente no se efectúa adecuadamente, lo que trae como consecuencia que no se logren los niveles de motivación y asimilación necesarios en el estudiante.

- La evaluación del estudio independiente no es sistemática, predominando la evaluación unidireccional, no facilitándose el desarrollo de una actitud crítica, constructiva hacia la realidad, que propicie un aprendizaje desarrollador.

En correspondencia con el carácter actualizado y contextualizado que exige el proceso de enseñanza-aprendizaje, así como de los enfoques y métodos activos que deben caracterizar la enseñanza en la actualidad, creemos que se precisa elaborar una alternativa didáctica interdisciplinar que favorezca el perfeccionamiento de la comunicación educativa en el proceso de enseñanza-aprendizaje en el proceso de formación de maestros y profesores, como una de las vías para estimular la gestión del conocimiento de los estudiantes y a la vez reforzar la calidad de facilitador del profesor.

\section{Métodos}

La investigación acción participativa: como método combina la investigación orientada a la formación profesionales de perfil pedagógico y por la participación dentro del grupo objeto de estudio, lo que permitió ahondar en las principales cuestiones culturales y comunicativas de los mismos.

Análisis de documentos: a través del cual se toman en cuenta en la elaboración de la propuesta de las acciones de formación cultural y en pos de las competencias comunicativas.

La Tormenta de ideas: en función de propiciar el conocimiento de las formas de expresión oral y su sustento cultural como meta principal la expresión libre de los sentimientos, criterios y actitudes. 
Técnica para evaluar estilo comunicativo. (Reinoso y otros, 2004) en función medir la autovaloración en torno a la participación desarrollada en el acto comunicativo y su actitud hacia los demás.

\section{La comunicación educativa y el aprendizaje.}

La significación de la comunicación en los contextos educativos es de importancia decisiva, en tanto favorece el papel formativo que juega la misma en el logro de los objetivos educativos. La misma es a su vez, parte esencial en función del encargo social de las universidades, que como instituciones deben priorizar un intercambio que integralmente propicie y favorezca una interacción su educativa para con sus estudiantes. Los abordajes y experiencias didácticas que se desarrollan desde la enseñanza de las disciplinas; están llamados favorecer las prácticas comunicativas a partir del enfoque histórico-cultural como fundamento teórico-metodológico de la comunicación educativa.

\subsection{Papel y lugar de la comunicación educativa en el contexto didáctico.}

La comunicación es un proceso de intercambio entre un emisor y receptor que no recibe sin interactuar, en tanto el mismo se convierte en emisor. La etimología del concepto "comunicación" tiene su raíz en la palabra latina comunicare, que significa poner en común y constituye en consecuencia, una forma de expresión diversificada de los seres humanos que permite a través de sus diferentes formas de concreción, socializar ideas, teorías, deseos e impresiones. La comunicación es aprendida y aprehendida como necesidad a través de la relación del hombre con su medio social.

Entre los primeros sociólogos en estudiar el proceso de comunicación humana desde un enfoque sistematizado, Mead G. (1977), autor del llamado "interaccionismo simbólico". La interpretación que propone este autor acerca del proceso comunicativo, deriva de entender la naturaleza social del individuo: es decir, plantea que la persona se forma en interacción con otros, mediante un proceso a través del cual se establece el control de sus acciones, gracias a la representación que acerca de ella se han conformado los seres humanos que le rodean.

Sin embargo, la notable significación de esta teoría para los estudios contemporáneos de la comunicación, ya que ha sido fuertemente censurada por estudiosos de orientación marxista, la misma sobrevalora el papel de

1 El Interaccionismo simbólico es una de las corrientes de pensamiento microsociológica, de gran incidencia sobre los medios, que está relacionada con la psicología social y la antropología, comprendiendo a la sociedad a partir de la comunicación. Como enfoque se ubica en los marcos del paradigma interpretativo que entiende el sentido de la acción social desde la perspectiva de los participantes y que concibe a la comunicación como una producción de sentido dentro de un universo simbólico determinado. 
los símbolos en la explicación de la conducta humana, e ignora la influencia de todo el conjunto de relaciones sociales y de la cultura en su totalidad, sobre el comportamiento, al analizar la interacción aislada que los individuos protagonizan a través de su actividad humana.

Sin lugar a dudas, de todos los sistemas de signos conocidos el más perfecto es la lengua, formado por las palabras, que constituyen signos verbales o lingüísticos, que les permiten a las personas referirse a la realidad, aún cuando aquello de que se hable no se encuentre presente.

El lenguaje oral es el principal sistema de comunicación decisivo para el trabajo docente. El lenguaje escrito trata de ser una aproximación, mediante unos signos gráficos, de lo que decimos hablando. Si se tiene en cuenta que cada disciplina tiene su propio lenguaje, entonces el docente de la disciplina en cuestión, tiene que estar preparado para comunicarse con sus estudiantes en ese lenguaje y ser capaz de que este llegue a utilizarlo de forma eficiente en su labor como futuro docente.

La comunicación oral y la comunicación escrita poseen semejanzas y diferencias (León, 2012:22):

- "Que la comunicación oral es inmediata en el tiempo; se produce directamente mediante la relación interpersonal; se apoya en los códigos no verbales (los gestos, la postura, el movimiento del cuerpo, etc.); se enmarca en un contexto real; admite digresiones, saltos temáticos, redundancias; aparecen reiteraciones léxicas, onomatopeyas, muletillas y frases hechas; la información compartida previamente es muy relevante, por lo que son frecuentes las oraciones inconclusas, las omisiones, el desorden de los elementos de la oración.

- Que la comunicación escrita traspasa los límites del espacio y el tiempo; al no estar presente el receptor, la interacción no es inmediata o no llega a producirse nunca y, generalmente, el que escribe no tiene certeza de quienes leerán el texto, por lo que se dice, con sobrada razón, que la palabras están solas en el texto escrito. Este se ajusta con rigor a las reglas gramaticales que garantizan la coherencia textual. Su estructura sintáctica es compleja y posee mayor riqueza léxica y precisión."

El proceso comunicativo depende del contexto en que se desarrolla, del tipo de relaciones sociales e interpersonales que se desarrollan entre sí y, por del grado de preparación y disposición volitiva consciente, que el conjunto de estos ingredientes socio-contextuales y los propios rasgos de la personalidad de los individuos que interactúan, otorguen a la cualidad y calidad del proceso comunicativo en líneas generales.

En el contexto educativo, la comunicación educativa es el elemento portador de toda la información cultural de los programas y textos de estudio, que requiere por un lado, de un guía interpretador de las esencias del conocimiento (el profesor) y por otro lado, de perceptores activos y comunicativos (los estudiantes). Ellos reinterpretan y asimilan conscientemente los contenidos recibidos, para convertirlos en acciones futuras concretas y aportan cambios al propio conocimiento y a su entorno social, a partir del enriquecimiento cognoscitivo de las diferentes formas de la actividad humana que el hombre ejecuta en su devenir cotidiano. 
La comunicación educativa, así entendida, ha transitado a nivel universal por un largo camino de evolución histórica, desde Comenius ${ }^{2}$ y de teorías contemporáneas acumuladas durante siglos, permitiendo tomar como punto de referencia acertado, los fundamentos dialéctico-materialistas y humanistas del Enfoque Históri$\mathrm{co-Cultural}^{3}$, para encarar con objetividad el proceso comunicativo de la educación y, en particular, para abordar sus especificidades y complejidades en la educación superior contemporánea.

Las responsabilidades y funciones de la universidad contemporánea redimensionan el papel del profesor, pero en ocasiones llega a obviarse la necesidad de precisar y, más aún, de re-conceptualizar su función básica, ante todo, buenos comunicadores y delineadores de una cotidianidad de estilo de vida universitario (Fernández, Durán y Álvarez, 1995).

Es así como se comprende, que la comunicación educativa contemporánea debe ser replanteada al interior de sus fundamentos y en su modus operandi, de manera diferente (Aparicio, 2003). Al respecto ocurre que, con demasiada frecuencia, se olvida que, entre lo que se decide al respecto en los altos niveles del diseño curricular y en las decisiones administrativo-institucionales, y lo que ocurre en la práctica concreta de la base, o sea en el proceso formativo integral y de enseñanza-aprendizaje puede mediar, y de hecho, media, una considerable distancia.

Por ello es necesario, trazar acciones tendientes a orientar y fortalecer el funcionamiento comunicativo de profesores y estudiantes, en particular, dentro de su núcleo presencial.

\subsection{El sistema de clases como componente interactivo presencial: necesidad de su perfeccionamiento comunicativo.}

Las formas de organización del proceso de enseñanza-aprendizaje caracterizan la dinámica de los diferentes modelos pedagógicos de formación (presencial, semipresencial y a distancia), ya que en ellos impactan de manera creciente el vertiginoso desarrollo de la Ciencia y la Tecnología, modificando desde el punto de vista didáctico los procederes de profesores y estudiantes y cómo se conciben los demás componentes del proceso de enseñanza-aprendizaje.

2 Jan Amos Komensky, en latín Comenius, nació el 28 de marzo de 1592 y murió en Ámsterdam el 15 de noviembre de 1670, sentó los fundamentos de la educación para todos los hombres en su Didáctica Magna, contribuyendo a crear una ciencia de la educación y una técnica de la enseñanza que aún perdura hoy día.

3 El enfoque histórico cultural representado por Vygotsky y sus seguidores, reconoce de forma particular la importancia de la actividad humana y su trascendencia social. Considera el aprendizaje como una actividad de índole social, caracterizando el carácter productivo y reproductivo del conocimiento, mediante la cual son asimilados los modos sociales de acción e interacción, reconociendo el activismo del sujeto en interacción subjetiva en su relación para con el objeto que acondiciona su desarrollo y evolución psíquico- física. 
La particularidad de diseñar y organizar el proceso para enseñar a un grupo de personas, hace que el conocimiento tenga un mayor alcance y "productividad" y que el docente se vea en la necesidad de demostrar la calidad de su preparación, el nivel científico de sus conocimientos y las habilidades didácticas que posee para trasmitir, y que los estudiantes se apropien de los hábitos de estudio y de los métodos de trabajo que emanan de su quehacer conformando un "patrón" del cual se nutren todas aquellas personas que perciben su influencia educativa.

La clase constituye la forma fundamental de organización del proceso de enseñanza-aprendizaje en todos los niveles de educación. Debe lograrse la unidad entre lo instructivo, lo educativo y lo desarrollador (Pérez Sibila, 2012) y otros aspectos de gran importancia como la comunicación interpersonal efectiva, el trato afable, el respeto mutuo y la tolerancia, el leguaje adecuado, la presencia personal, el clima pedagógico adecuado y emocionalmente compatible, así como eliminar tratamientos discriminatorios e injustos a los estudiantes.

\subsection{Retos de la comunicación educativa en el sistema de clases: necesidad de su carácter interdisciplinario.}

En el modo de actuación profesional del maestro, la comunicación interpersonal es el instrumento fundamental. Esta debe estar provista de una fuerte base cognitiva y sociocultural sobre el proceso de desarrollo de la personalidad integral del individuo y de su manifestación y desafíos, a través de las diferentes actividades de socialización humanas.

La comunicación educativa tiene una función especialmente importante, particular y compleja para el futuro egresado de las carreras pedagógicas:

- Es un componente didáctico esencial de la formación integral del futuro educador, y debe estar dirigida no solo a transmitir conocimientos básicos y a orientar métodos para su adquisición, sino también, debe desarrollar las propias habilidades profesionales de comunicación interpersonal para el tratamiento de problemas de la personalidad y del comportamiento humano.

- El propio perfil profesional del educador en formación, necesita establecer nexos verbales lógicos y continuos entre el contexto social más amplio y los diversos contextos grupales como objetos de estudio y de trabajo.

- Por la necesaria naturaleza interdisciplinar e integradora que el desarrollo de la base cultural y técnica específica que este modo de actuación integrador requiere en la formación del futuro egresado.

- No debe olvidarse que el educador es, ante todo, un profesional de la comunicación-orientadora interpersonal y que dicha formación comunicativa especializada, debe ser modelada interactivamente ante, 
con, y entre los estudiantes según Vigotsky ${ }^{4},(1934)$, bajo la dirección y orientación participativa del profesor, al menos en los aspectos esenciales de sus múltiples formas posibles y variadas de construcción y desarrollo.

Ante los requerimientos específicos ya planteados como imprescindibles para la formación profesional del educador, no basta limitar el espacio del sistema de clases a servir de orientación didáctica para el estudio independiente grupal o individual, del estudiante. Además, es necesario integrar en estos espacios componentes de modelación comunicativa profesional a todas las disciplinas que forman los planes de estudios.

\section{Relación entre la comunicación educativa y la interdisciplinariedad para el perfeccionamiento del sistema de clases}

El elemento esencial de la interdisciplinariedad está dado por los nexos o vínculos de interrelación y de cooperación entre disciplinas sobre la base de objetivos comunes. Esa interacción hace aparecer nuevas cualidades integrativas, no inherentes a cada disciplina aislada, sino a todo el sistema que conforman y que conduce a una organización teórica más integrada de la realidad.

La interdisciplinariedad adquiere una connotación especial para la práctica pedagógica, debe constituir un principio rector para el diseño y desarrollo de los currículos, en particular para la formación de profesores.

Del estudio realizado se concluye que existen aspectos básicos:

1. Para que exista interdisciplinariedad, debe existir primero una realidad multidisciplinar evidente, que participe en un esfuerzo común de formación hacia un objetivo determinado.

2. Es a partir de la base común, que se comprende que el primer esfuerzo por transformar a integrar conocimientos, etc. comienza con el concepto de interdisciplinariedad, como estrategia o proceso de interrelación de campos del saber humano, a través de diferentes formas de la actividad, y expresando esta interrelación mediante la propia formulación y desarrollo de las categorías didácticas fundamentales que rigen el proceso formativo integral.

3. De esta relación interdisciplinar de partida en lo curricular del proceso de enseñanza-aprendizaje y de formación integral del futuro profesional, se aspira a promover y alcanzar el desarrollo de un desempeño

4 Lev Semiónovich Vigotsky, ruso, nace el 17 de noviembre de 1896, en Orsha, actual Bielorrusia y muere 11 de junio de 1934. Su concepción parte de considerar que el desarrollo de los humanos únicamente puede explicarse en términos de interacción social, a través de instrumentos culturales (como el lenguaje), que es resultado y trasmisor de la cultura a través de la interacción social, su entendimiento de la misma, asume un papel predominante en su teoría. 
progresivamente transdisciplinar integrador, que se expresa, finalmente, en el propio modo de actuación profesional del futuro egresado.

Para ello, la comunicación educativa, además de ser instrumento central en la trasmisión de los conocimientos necesarios; es también espacio de modelación de la fundamental herramienta de interacción profesional con la que cuenta el educador para su desempeño en todos los perfiles en los que se desenvuelva.

\section{El enfoque histórico-cultural como fundamento teórico-metodológico de la comunicación educativa para el siste- ma de clases de una disciplina.}

Se considera el papel de la comunicación educativa como herramienta insuperable en manos del profesor, en las condiciones de una zona de desarrollo próximo, que, para el caso del sistema de clases de una disciplina, se manifiesta en un espacio interactivo de nuevo tipo, en la que el elemento de tiempo y espacio compartidos sistemáticamente por profesores y alumnos, se cambia, por una relación espacio-temporal más mediatizada.

Al respeto, el enfoque vigotskiano sostiene que las acciones de aprendizaje ocurren siempre en dos planos:

Uno externo, socializado, interpersonal, determinado a partir de la interrelación dialógica entre el profesor y sus estudiantes, y entre estos, actuando sobre el objeto de aprendizaje, el que contiene a su vez, en síntesis, un determinado volumen de conocimientos culturales acumulados por la humanidad en su desarrollo histórico.

Luego, en el plano interno, esas mismas acciones de aprendizaje se reeditan progresivamente, de manera individual e interpersonal, mediante un proceso de consciente aprehensión y asimilación de los contenidos.

En todo éste proceso, el lenguaje humano desempeña un papel fundamental, constituye el elemento que vincula la información objetiva y subjetiva acerca del objeto de aprendizaje y las propias impresiones y transformaciones psicológicas que el individuo experimenta. Al transformar la realidad como ejercicio formativo, transformándose a su vez a sí mismo, en el transcurso de la actividad cognoscitiva.

En la formación integral de la personalidad, a través de la relación social que los seres humanos establecen en la actividad humana, subyace el concepto vigotskiano de "zona de desarrollo próximo", de plena vigencia en las nuevas condiciones de prioridad del autoaprendizaje y el autodesarrollo.

5 La zona de desarrollo próximo (ZDP) denota la brecha entre las habilidades que posee el/ la niño(a) y a lo que pueden llegar a aprender con la guía de un adulto o de un par más competente, se basa esencialmente en la relación entre las habilidades actuales del niño y su desarrollo potencial. 
Son estos los principios del carácter histórico y culturalmente determinado del proceso de enseñanza-aprendizaje; el principio del carácter objetal del proceso de enseñanza-aprendizaje; el principio del carácter desarrollador del aprendizaje y el principio del carácter científico del mismo.

Sobre esta base de análisis, la concepción general del modelo de comunicación educativa, debe contemplar, ante todo, las exigencias del desarrollo integral de la personalidad del estudiante universitario en los nuevos contextos, y precisar qué demandas plantean estas exigencias al proceso comunicativo.

\section{La alternativa didáctica: sus fundamentos.}

El término alternativa, presupone la existencia de una disyuntiva o encrucijada a partir de un dilema existente.

Una alternativa didáctica es "una vía, forma o procedimiento para la dirección del proceso de enseñanza-aprendizaje (PEA), que se distingue de otras con objetivos y/o propósitos iguales o similares, en atención a su singularidad. Ella representa una variante contextualizada, que constituye una opción a escoger para la planificación, organización, regulación, control y/o evaluación del PEA”(Ballester, S., 2009, p.5).

A partir de lo expresado por Ballester, S. (2009), en esta investigación se contextualiza que una alternativa didáctica para favorecer la interdisciplinariedad, y en particular, la comunicación educativa en la formación del profesor, es:

La opción para dirigir el proceso de enseñanza-aprendizaje, dentro de otras variantes didácticas, en la que participan activamente profesores y estudiantes en una integración armónica para favorecer la interdisciplinariedad, y en particular, la comunicación educativa en el proceso de enseñanza-aprendizaje de una disciplina y su enseñanza, para la formación de profesores, a partir de las potencialidades que brinda el diseño del currículo.

\section{Síntesis de la alternativa propuesta. Una visión Algorítmica, (Anexo 1).}

Como parte de los fundamentos filosóficos, la alternativa tiene como sustento la concepción dialéctico materialista como metodología universal del conocimiento científico. Los principios de la dialéctica materialista constituyen de hecho, fundamentos básicos de la alternativa didáctica, dentro de los que se tienen en consideración el principio de la objetividad, dada la relación que se establece entre la propia propuesta y la realidad en virtud de la necesidad de favorecer la interdisciplinariedad, y en particular, la comunicación educativa en el proceso de enseñanza-aprendizaje.

El principio de la concatenación universal avala las relaciones que se establecen entre los componentes de la alternativa, las que son resultado del reflejo de las que se producen en el proceso de enseñanza-aprendizaje de 
una disciplina en cuestión y al tratar de favorecer la interdisciplinariedad y las relaciones interdisciplinarias a través de una eficiente comunicación educativa.

Como parte de los fundamentos sociológicos, la alternativa se fundamenta en las demandas actuales de la sociedad de lograr una enseñanza que, si bien mantiene la concepción curricular por disciplinas y asignaturas, se pronuncia en contra de la fragmentación del conocimiento y por su transversalización y pretende revelar la función social del conocimiento, a partir de la estrecha relación existente entre ciencia-tecnología- sociedad y medio ambiente, y a través de la vinculación entre la institución formativa, la familia, la comunidad y sus organizaciones.

Desde el punto de vista psicológico la alternativa didáctica en cuestión se sustenta en el "enfoque histórico cultural" formulado por L. S. Vigotsky y sus seguidores, relacionado con el desarrollo de la psiquis y la personalidad a partir del condicionamiento histórico-social. Enfoque que considera de vital importancia el papel de la mediación en el proceso de apropiación de la cultura.

Los significados históricos - culturales heredados, mediados a través del contexto, las herramientas y los signos son reconfigurados o resignificados por los estudiantes, que les atribuyen sentido al vincularlos con su mundo cognitivo, experiencial y afectivo.

En lo pedagógico, la alternativa didáctica toma en consideración el carácter humanista y transformador de la pedagogía, en este sentido la interdisciplinariedad y la comunicación educativa. En su calidad de principio metodológico favorece el carácter educativo, formativo y transformador del proceso de enseñanza-aprendizaje, en tanto la cooperación y transversalización de las diferentes asignaturas se ha de alcanzar sobre la base de la unidad entre lo instructivo, lo educativo y lo desarrollador.

La interdisciplinariedad y la comunicación educativa, contribuyen a lograr la formación integral del estudiante en tanto:

- Las asignaturas que se relacionan en dicho proceso logran que los estudiantes comprendan la sociedad y el mundo globalizado que les ha tocado vivir.

- Se puedan orientar ante contingencias e intervenir de forma constructiva como factores de cambio guiados por una autonomía competente y responsable, basada en valores para con su entorno social y natural.

- Aportan elementos importantes que garantizan la formación de convicciones, principios, valores, normas de comportamiento y un modo de actuación profesional pedagógica, acorde con el ideal de hombre que se aspira formar.

El proceso de enseñanza-aprendizaje de una disciplina y su enseñanza en la formación de profesores, desde una perspectiva interdisciplinaria, basada en la eficiencia de la comunicación educativa, propicia la coopera- 
ción, la solidaridad, el respeto al criterio ajeno a partir de las propias tareas de búsqueda, indagación, contribuyendo de esta manera a la socialización de aquello que ha sido buscado en otras fuentes de información. La potenciación del trabajo en equipos, hace que el estudiante crezca espiritualmente a partir de lo que le aporta la integración y sistematización de los contenidos y por tanto pueda transferirlo a otras asignaturas.

Concebir el proceso de enseñanza-aprendizaje hacia la búsqueda activa del conocimiento significa lograr que el estudiante establezca las relaciones entre los diferentes contenidos que ha de asimilar a partir de las diferentes asignaturas que forman parte del currículo. Estas relaciones esenciales han de encontrar su concreción en la realización de tareas que requieran de la integración y sistematización de diferentes contenidos y de la consulta de diversas fuentes del conocimiento, y reconocer, el valor de los procesos lógicos del pensamiento en la determinación de los elementos del objeto de estudio, así como el empleo de procedimientos didácticos que potencien en el profesor en formación la capacidad para resolver tareas en las que se requieran analizar, sintetizar, generalizar, comparar, abstraer y concretar, así como aplicar capacidades cognitivas diversas.

TABLA 1.

\section{Síntesis comparativa de los componentes de la alternativa didáctica interdisciplinar para el perfeccionamiento de la comunicación educativa en el sistema de clases de una disciplina y su enseñanza.}

\begin{tabular}{|c|c|}
\hline Actual & Propuesta \\
\hline \multicolumn{2}{|c|}{ Objetivos de la comunicación educativa } \\
\hline $\begin{array}{l}\text { Orientar, retroalimentar y evaluar las invariantes del } \\
\text { contenido de una Disciplina y su Metodología. No se } \\
\text { cumple con el carácter interdisciplinar previsto en los } \\
\text { programas, ni se utiliza una adecuada estrategia comu- } \\
\text { nicativa en cada una de las clases. }\end{array}$ & $\begin{array}{l}\text { Además debe develar la relación integradora inter y transdis- } \\
\text { ciplinar entre las disciplinas de la carrera, en función de una } \\
\text { formación sólida del modo de actuación comunicativo-profe- } \\
\text { sional del futuro profesor, pero desde matices propios y espe- } \\
\text { cíficos, partiendo de un núcleo conceptual común, al asumir } \\
\text { la comunicación de forma clara y precisa con la ayuda de la } \\
\text { terminología y simbología de cada disciplina de manera oral, } \\
\text { escrita y/o visual. }\end{array}$ \\
\hline
\end{tabular}




\section{Contenidos de la comunicación educativa}

El núcleo discursivo que explica y resume la base orientadora de la acción (BOA) relativa a los contenidos de la disciplina, para el desarrollo de las acciones y operaciones del sistema de tareas de aprendizaje, a través del trabajo independiente colectivo e individual en esa asignatura y disciplina en específico.

\section{Métodos para el perfeccionamiento de la comunicación educativa}

La exposición conceptual por parte del profesor, en interacción con los estudiantes, de los núcleos discursivos de orientación, de retroalimentación y de evaluación de los contenidos al interior de la disciplina, pero siempre combinando, elementos de orientación con nuevos contenidos, retroalimentación del proceso de aprendizaje en desarrollo y evaluación del mismo, pero sin relacionarlos de manera intencional a través de un enfoque interdisciplinar durante el desarrollo del proceso de enseñanza-aprendizaje en el sistema de clases.
Este núcleo discursivo en su interrelación sistémica con las invariantes de contenido en otras disciplinas de la carrera, para propiciar un proceso de aprendizaje integrador inter y transdisciplinar en correspondencia con el modo de actuación del futuro profesor; además se ilustren los rasgos comunes y particulares del tratamiento del contenido, sus relaciones de semejanza, interrelación y diferencia, trabajando de forma tal que primero se logre de lo general a lo particular, para después ir a lo específico de la disciplina, y regresar de lo particular, de nuevo a lo general enriquecido, de forma tal que el futuro profesor se apropie de la dinámica del trabajo en el aula
La utilización de los métodos de la enseñanza problémica en particular la exposición problémica, la búsqueda parcial y el método investigativo, sobre la base de cómo deberá ser impartido este contenido por este profesional en su futuro accionar como profesor y en el que la heurística juegue un papel fundamental, sobre todo, con la utilización de los principios heurísticos. Para ello la comunicación educativa juega un papel decisivo y se refuerzan las relaciones inter y transdisciplinares.

Además, según la organización por cada clase dentro del sistema, la primera y segunda clase se emplean para presentar a los estudiantes las invariantes de conocimiento y la Base Orientadora de la Acción (BOA) de toda la asignatura, con sus nexos inter y transdisciplinares y con las invariantes de contenido de otras disciplinas de la carrera. En las restantes clases dentro del sistema se utiliza el mayor tiempo para debates, análisis, etc. en los que se ampliará el tratamiento problematizado inter y transdisciplinar de situaciones conflictivas, etc. frecuente en el desempeño profesional, propiciando así mayor espacio para la activación de los nexos interactivos en el espacio del aula y fuera de esta. 
Medios de apoyo a la comunicación educativa y ambientes de aprendizaje.

Medios tradicionales (pizarrón, tiza, libros de textos, materiales impresos, guías de estudio, software educativo de apoyo con textos y materiales digitalizados, la propia voz del profesor y de los estudiantes, empleo ocasional de videos y filmes en otros soportes).
Los estudiantes podrán diseñar sus propios sistemas multimedia, que muestren y problematicen aplicaciones concretas del modo de actuación del educador en sus diferentes áreas de desempeño, y que permitan, en su combinación con guías y tareas de aprendizaje, una mejor explotación de las relaciones inter y transdisciplinares manifiestas en el modo de actuación del futuro profesional.

Diseño y empleo de mapas conceptuales, cuadros sinópticos integradores, tarjetas de aprendizaje, resúmenes de contenidos, gráficos, diagramas, y la revisión del diseño comunicativo de las guías de estudio vigentes para su perfeccionamiento y adecuación a una perspectiva integradora inter y transdisciplinar.

Síntesis de la comunicación educativa como componente activo en el proceso de evaluación.

Concretada en el diseño exámenes escritos, a nivel reproductivo
Diseño de un sistema de evaluación que combine la interacción escrita y oral con un diálogo fluido que permita al estudiante interactuar con su grupo y con el profesor y que prevea el planteamiento de problemas profesionales inter y transdisciplinares de complejidad creciente, para lo cual es posible aprovechar también el sistema multimedia de interacción comunicativa. Análisis y modificación del diseño de las evaluaciones escritaws, con el planteamiento de problemas abiertos a la reflexión, la interpretación del problema para la búsqueda de soluciones y que permita la elaboración de textos que integren contenidos de varias asignaturas. Además deben modificarse las forma de calificación, cuidando que no se diseñen claves de formulación cerrada para ejercicios cuyo tratamiento comunicativo y exposición discursiva puede ser válidamente diversa, desde diferentes puntos de enfoque y debiendo reflejar en su formulación una perspectiva inter y trans-disciplinar dados los objetivos formulados. 


\section{CONCLUSIONES}

- Los fundamentos teórico-metodológicos permitieron argumentar que el sistema de clases de la Educción Superior actual, necesita de mayor profundización en la investigación didáctica, para perfeccionar aspectos hoy insuficientes de su ejecución, los que no son superables solo a partir del trabajo docente y docente-metodológico.

- La comunicación educativa constituye un área necesitada de perfeccionamiento y remodelación, a partir de los retos actuales que impone la orientación del proceso de aprendizaje y la propia necesidad de interacción en el sistema de clases de una disciplina.

- El sistema de clases de una disciplina y su enseñanza requieren del perfeccionamiento de la comunicación educativa por la naturaleza compleja que reclama la formación del futuro profesional de una carrera pedagógica lo que corroboró el diagnóstico realizado que involucró a directivos, profesores y estudiantes.

- La comunicación educativa en una disciplina y su enseñanza, en la formación de profesores, permite el establecimiento de las relaciones interdisciplinares que deben establecerse, aprovechando para ello más eficaz y pertinentemente el espacio interactivo del sistema de clases, como condición necesaria para la integración de contenidos en el proceso de interiorización y consolidación del modo de actuación profesional del futuro profesor.

- La alternativa didáctica interdisciplinar para el perfeccionamiento de la comunicación educativa en el sistema de clases de una disciplina, se basó fundamentalmente en la búsqueda de la mayor pertinencia y adecuación de una nueva concepción integral para el tratamiento sistémico de las categorías didácticas del proceso, durante el desarrollo del sistema de clases de la disciplina, a través de una concepción interdisciplinar novedosa de la comunicación educativa en estos espacios de interacción presencial alumno-profesor-alumnos del grupo clase en general. 


\section{REFERENCIAS BIBLIOGRÁFICAS}

Aparicio, J. J. (2003). La psicología del aprendizaje y los modelos de diseño de enseñanza: la Teoría de la elaboración. Rev. Tarbiya, No 1-2. Julio-Nov. UAM, p.19.

Ballester, S. (2009). Alternativas didácticas para la formación del profesor de Ciencias Exactas en el área de Matemática. Evento Didáctica de las Ciencias. La Habana: UCP "Enrique José Varona”. Soporte digital.

Ballester, S. (2009) Ibidem

Fernández, A. M; Durán, A y Álvarez, MI. (1995): Comunicación Educativa. Editorial Pueblo y Educación. La Habana.

León Moya, M. (2012). Alternativa didáctica interdisciplinar para el perfeccionamiento de la comunicación educativa en el sistema clase-encuentro de los años terminales de la Licenciatura en Psicología. (Tesis de Maestría). Universidad Camilo Cienfuegos, Matanzas, Cuba.

Pérez Sibila, A. (2012). Proyecto docente para la clase \# 2 de la asignatura Fundamentos Biológicos del Comportamiento Humano. Sede Universitaria Jagüey Grande. Matanzas.

Vigotski, L. S. (1982). Pensamiento y lenguaje. La Habana: Editorial Pueblo y Educación. 


\section{BIBLIOGRAFÍA}

Artola, M. L.; Petersson, M. (2007). La semipresencialidad como tendencia en la educación superior cubana, una experiencia práctica. [CD-ROM]. Universidad 2008. CEDE. UMCC.

Ballester, S. (2009). Alternativas didácticas para la formación del profesor de ciencias exactas en el área de Matemática. Evento Didáctica de las Ciencias. La Habana: UCP “Enrique José Varona”. Soporte digital.

Bermúdez Sarguera, R. (2002). Teoría y metodología del aprendizaje. Ciudad de la Habana: Editorial Pueblo y Educación.

Bertalanffy, L.V. (1951): General system theory - A new approach to unity of science (Symposium), Human Biology, Dec 1951, Vol. 23, p. 303-361

Boza, Y. (2010). La preparación interdisciplinaria de los profesores: un ejemplo en el área de Ciencias Exactas de la Educación Media Superior. La Habana. Tesis en opción al título de Máster en Ciencias Exactas. UCP "Enrique José Varona”.

Bracho, M. (2008). El docente y la comunicación en el aula. Comunicación didáctica en el proceso de enseñanza aprendizaje. República Bolivariana Venezuela: Universidad de los Andes.

Carpintrous Pérez, L. y Rizo, C. (1998). Indicadores e investigación educativa (Primera Parte). Instituto Central de Ciencias Pedagógicas de Cuba.

Castellanos, D. y otros. (2002). Aprender y enseñar en la escuela. Una concepción desarrolladora. La Habana: Editorial Pueblo y Educación. 123 p.

Chomsky, Noam (1979): Language and Mind. MIT University Press.

Delors, Jacques (1998): La educación encierra un tesoro. Informe a la UNESCO de la Comisión por la Educación del Siglo XXI. UNESCO, Paris.

Fernández de Alaíza, B. (2000). La interdisciplinariedad como base de una estrategia para el perfeccionamiento del diseño curricular de una carrera de ciencias técnicas y su aplicación a la ingeniería en automática en la República de Cuba. Tesis de Doctorado. Instituto Superior Politécnico José Antonio Echeverría. La Habana. Cuba.

Fernández, A. M; Durán, A y Álvarez, MI. (1995): Comunicación Educativa. Editorial Pueblo y Educación. La Habana.

Fernández González, A. M. (2003) et al. Comunicación educativa. Ciudad de La Habana: Editorial Pueblo y Educación. 71-90p.

Galindo, O. (2007). El proceso de enseñanza aprendizaje desarrollador en condiciones de semipresencialidad. [CD- ROM. CIUM 2007. CEDE. UMCC. 
González A. M.; Recarey S. y Addine F. (2002). “Cap. 5: La dinámica del proceso de enseñanza- aprendizaje mediante sus componentes", Aprender es crecer. La Habana.

Horruitiner, P. (2008): La Universidad cubana; el modelo de formación. Editorial Félix Varela, La Habana.

Hymes, Dell (1992): On Communicative Competence. En. Pride, J.B. and Holmes, Janet (eds.) (First and second publications in 1967, 1976).

Jiménez, L. (2007). La interdisciplinariedad desde un enfoque profesional pedagógico: un modelo para el colectivo de año. Matanzas. Tesis de Doctorado. UCP "Juan Marinello Vidaurreta”.

León Moya, M. 2012. Alternativa didáctica interdisciplinar para el perfeccionamiento de la comunicación educativa en el sistema clase-encuentro de los años terminales de la Licenciatura en Psicología. Tesis en opción al Título de Máster en Ciencias de la Educación Superior. Matanzas.

Marcelo Rojas, C. (2002). Manual de Investigación y Redacción Científica. Universidad Alas Peruana. Lima. Perú.

Mead, G. (1977): Symbolic Interactions. New Library Line, London.

Merrill-Taylor, Wolfgang Charles (2009): Blending Higher Education through On-Line Chats. UT Press, University of Toronto. Toronto, Canada.

Milián, Y. (2010). La formación interdisciplinaria en el primer año de la especialidad Profesor General Integral de Secundaria Básica a través de la asignatura Matemática para la Secundaria Básica y su Metodología

I, Matanzas. Tesis en opción al grado científico de Máster. UCP “Juan Marinello Vidaurreta”.

Moll, L. (1990): Vigotsky and Education. Cambridge. University Press.

Perera, (2007): La comunicación educativa y sus nuevos desafíos en la Educación Superior cubana. (Consultado 12 junio 2011). En: www.revistaiberoamericanadeeducacion/oie/org

Perera, F. (2004 a). La práctica de la interdisciplinariedad en la formación de profesores. En Interdisciplinariedad: Una aproximación desde la enseñanza aprendizaje de las ciencias, Marta Álvarez Pérez (Comp.). La Habana: Editorial Pueblo y Educación.

Reinoso Cápiro, C. /s. a /. El proceso enseñanza-aprendizaje desarrollador y la comunicación interpersonal en el trabajo en colaboración. La Habana.

Reinoso Cápiro, C.; Rodríguez García, M. A.; Linares Cordero, M. (2004). "Una experiencia interesante: mi comunicación con los demás y conmigo mismo”. En García Batista, G (comp.). Temas de introducción a la formación pedagógica. La Habana: Editorial Pueblo y Educación. 
Rodríguez Morell, J. L. (2011). Toward the development of a metacognitive intercultural communicative competence in the education of students of interpreting: general theoretical/ pragmatic foundations (I) En: International Journal of Translation \& Interpreting, Vol.3 (No.1). Science Citation Index. Base Ulrichs. Recuperado de: http://www. trans-int.org.com

Sánchez, E. (2013): Alternativa didáctica interdisciplinar para el perfeccionamiento de la comunicación educativa de la Matemática en la carrera de Licenciatura en Educación Primaria. Tesis en opción del Título Académico de Máster en Matemática Educativa. Matanzas.

Sinclair, Lewis (2004): Learning to learn far away from the classroom. McCormick Editors. London.

Torres, J. (1994). Globalización e interdisciplinariedad: el currículum integrado. Madrid: Editorial Morata.

UNESCO (1998): Declaración mundial sobre la Educación Superior en el siglo XXI. UNESCO Press, París.

Vigotsky L. S. (1987). Historia del desarrollo de las funciones psíquicas superiores. Editorial Científico Técnica. La Habana.

Zilberstein, J. y Silvestre, M. (2004). Didáctica desarrolladora desde el enfoque histórico cultural. Ediciones CEIDE. 


\section{ANEXOS}

Alternativa didáctica para el perfeccionamiento de la comunicación educativa en el proceso de enseñanza-aprendizaje de una disciplina y su enseñanza, para lo estudiantes de formación pedagógica
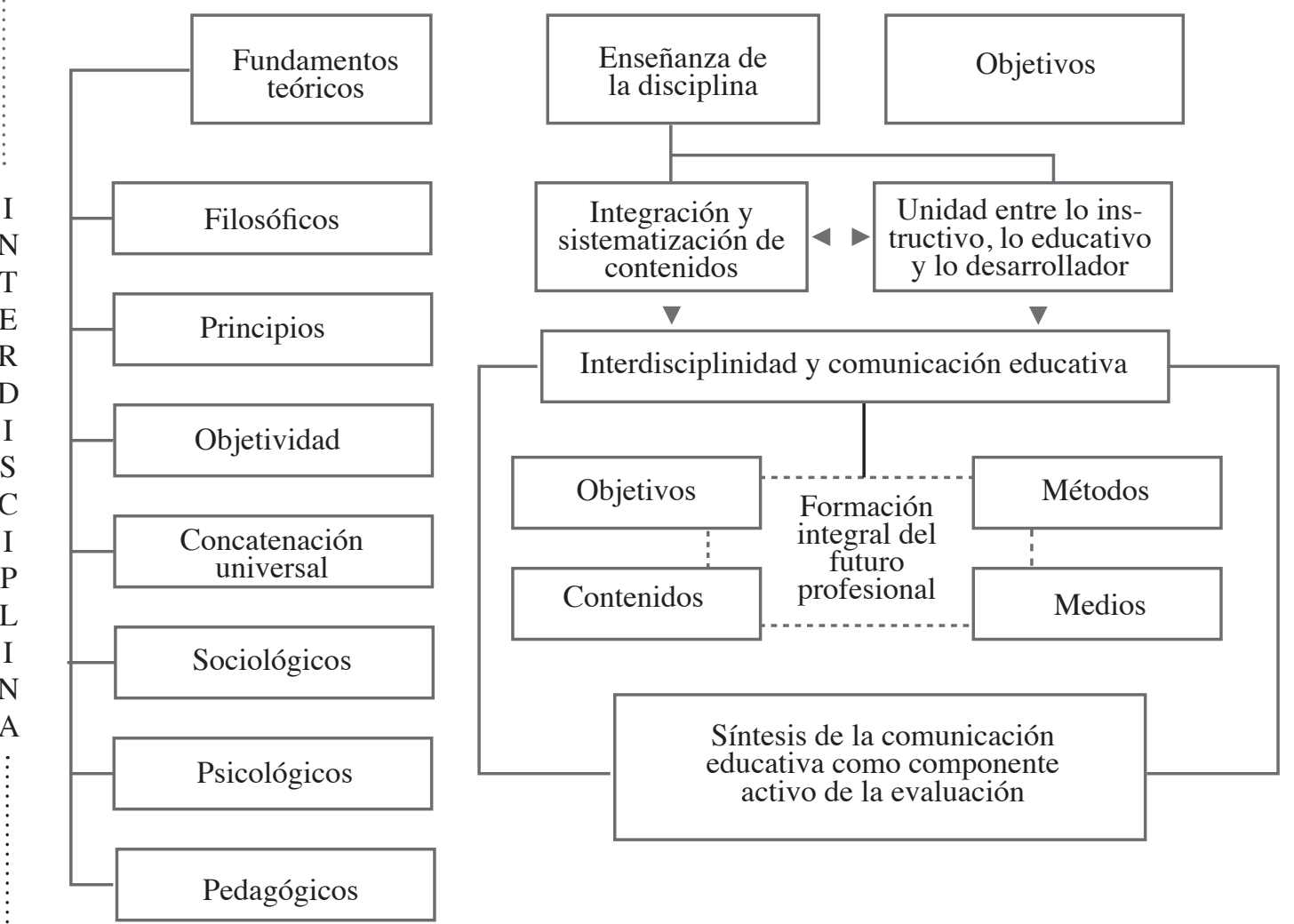
de un disciplina y su enseñanza en la formación de profesores 